\title{
Molecular Characterization of a Reemergent Brugia malayi Parasite in Sri Lanka, Suggestive of a Novel Strain
}

\author{
C. H. Mallawarachchi ${ }^{1},{ }^{1}$ T. G. A. N. Chandrasena $\left(\mathbb{D},{ }^{2}\right.$ G. P. Withanage, ${ }^{2}$ R. Premarathna $(\mathbb{D})^{2}$ \\ S. M. N. S. M. Mallawarachchi $\mathbb{D}^{3},{ }^{3}$ Nilmini Y. Gunawardane $\mathbb{D}^{2},{ }^{2}$ Ranil S. Dasanayake $\mathbb{D}^{4},{ }^{4}$ \\ Dinesh Gunarathna $\mathbb{D}^{5},{ }^{5}$ and N. R. de Silva $\mathbb{D}^{2}$ \\ ${ }^{1}$ Medical Research Institute, Colombo, Sri Lanka \\ ${ }^{2}$ Faculty of Medicine, University of Kelaniya, Ragama, Sri Lanka \\ ${ }^{3}$ Ministry of Health and Indigenous Medicine, Colombo, Sri Lanka \\ ${ }^{4}$ University of Colombo, Colombo, Sri Lanka \\ ${ }^{5}$ Ministry of Plantation Industries, Battaramulla, Colombo, Sri Lanka
}

Correspondence should be addressed to C. H. Mallawarachchi; harendra9791@gmail.com

Received 21 March 2021; Accepted 28 July 2021; Published 10 August 2021

Academic Editor: Khalid Mehmood

Copyright (C) 2021 C. H. Mallawarachchi et al. This is an open access article distributed under the Creative Commons Attribution License, which permits unrestricted use, distribution, and reproduction in any medium, provided the original work is properly cited.

\begin{abstract}
Sri Lanka achieved elimination status for lymphatic filariasis in 2016; still, the disease remains a potential public health issue. The present study is aimed at identifying a subperiodic Brugia sp. parasite which has reemerged in Sri Lanka after four decades via molecular-based analysis. Polymerase chain reaction performed with pan-filarial primers specific for the internal transcribed spacer region-2 (ITS-2) of the rDNA of Brugia filarial parasites isolated from human, canine, and feline blood samples yielded a $615 \mathrm{bp}$ band establishing the species identity as Brugia malayi. Comparison of the ITS2 sequences of the reemerged $B$. malayi isolates with GenBank sequences revealed a higher sequence homology with B. pahangi than B. malayi with similar phylogenetic evidence. However, the mean interspecies Kimura-2-parameter pairwise divergence between the generated Brugia sequences with $B$. malayi and B. pahangi was less than $3 \%$. During the analysis of parsimony sites of the new ITS2 sequences, substitutions at A36T, A296G, T373A, and G482A made the sequences different from both B. pahangi and B. malayi suggesting the possibility of a new genetic variant or a hybrid strain of $B$. malayi and B. pahangi. Mosquito dissections and xenomonitoring identified M. uniformis and M. annulifera as vectors of this novel strain of B. malayi circulating among cats, dogs, and humans in Sri Lanka.
\end{abstract}

\section{Introduction}

Lymphatic filariasis (LF) is a neglected tropical disease estimated to have affected 40 million people worldwide, with an at-risk population of 893 million people residing in 49 endemic countries [1]. Although infection is not fatal, it is a leading cause of permanent disability and was targeted for elimination as a public health problem by 2020 [1].

Three species of filarial worms, namely, Wuchereria bancrofti, Brugia malayi (nocturnally periodic strain), and Brugia timori, are known to cause classical lymphatic filaria- sis in humans. Of the three human filarial parasites, $W$. bancrofti is the most prevalent, causing $90 \%$ of infections worldwide, while the rest are attributed to B. malayi (prevalent in Southeast Asia and in South-Western parts of India) and to a lesser extent B. timori (limited to the islands of Timor-Leste in eastern Indonesia) [1]. A zoonotic strain of B. malayi (subperiodic strain), which is a natural parasite of a variety of wild and domestic animals (cats, dogs, monkeys, and slow lorises), has been documented to cause accidental zoonotic infections in humans [2-5]. Serological and molecular evidence of human infections by another zoonotic 
filarial worm, B. pahangi (a natural parasite of felines), has been reported from Kuala Lumpur, with clinical manifestations consistent with LF [6]. These zoonotic filariae (subperiodic B. malayi and B. pahangi) also contribute to the human disease burden especially in United States, South America, and Southeast Asian region [2, 3, 7].

Both $W$. bancrofti and B. malayi (nocturnally periodic strain) were prevalent in Sri Lanka in the past [8]. Vector control activities focused on clearance of aquatic vegetation (a requirement for breeding of Mansonia sp. mosquitoes) resulted in clearance of $B$. malayi infections from the country in late nineteen sixties [8]. Subsequently, five rounds of single annual mass drug administration (MDA) were implemented from 2002 to 2006 in the three provinces (Western, NorthWestern, and Southern) endemic for Bancroftian filariasis using diethylcarbamazine citrate $(300 \mathrm{mg})$ combined with albendazole (400 mg). In 2016, Sri Lanka was categorized as a country that had eliminated LF as a public health problem, following fulfilment of critical criteria stipulated by the World Health Organization (WHO) for the verification of elimination [9]. However, surveillance activities during the phases of post-MDA and postelimination revealed the sporadic occurrence of brugian filariasis in all three LF endemic provinces [10-12]. Periodicity studies revealed that the reemergent Brugia species microfilaria (MF) exhibited nocturnal subperiodicity, implying a strain of different origin [12]. Zoonotic surveillance in areas affected by human brugian filariasis has revealed a high prevalence of B. malayi microfilaraemia among dogs and cats [13]. The existence of another zoonotic filarial species, Brugia ceylonensis, among local canines has also been documented in the past [14].

Exact speciation of the reemerged Brugia parasite was important as zoonotic B. malayi, B. pahangi, and B. ceylonensis have all been implicated in accidental human infections $[3,4,6,15]$. The morphological similarity of MF of Brugia sp. necessitated a molecular-based approach (PCR and sequencing) for species identification [15].

Studies on the genetic variability of $B$. malayi indicate that parasite isolates from different regions diverged significantly [16]. Gene sequences of zoonotic Brugia species are sparse in major data bases, and with increasing human infections, there will be an increased need for genomic repositories [17]. Such information may support the genus or species clarifications based on consensus gene targets. Thus, sequencing of the Brugia sp. MF that were isolated from humans and animals of Sri Lanka was carried out to construct the phylogeny and assess their relationship to other Brugia species.

Knowledge of the transmission dynamics of the newly emerged parasite is important for control. The vector preferences of the reemerged parasite may differ from the nocturnal periodic strain of $B$. malayi documented in the past; thus, entomological investigations were performed for clarification of the vector species.

Against this background of events, a comprehensive analysis of the reemerged Brugia sp. parasite of Sri Lanka, as to its species identity, the phylogeny and genetic relatedness to other Brugia species and its vector preferences were studied using parasitological and molecular methods.

\section{Methods}

2.1. Human Sample Collection and DNA Extraction. A total of seven human blood samples positive for Brugia sp. MF by thick night blood smears (NBS) during the study period were included to the study. Positive samples were collected from four districts (Gampaha, Kalutara, Puttalam, and Galle) of the three endemic provinces, and one was from a case detected from a nonendemic district in the North Central province (Anuradhapura) (Figure 1). Nine blood samples positive for Brugia antibodies by the Brugia rapid test that were detected during an initial survey were also included in the molecular analysis [12]. The samples were stored at $-20^{\circ} \mathrm{C}$ in EDTA prior to DNA extraction. The MF in blood was concentrated by Nuclepore ${ }^{\circledR}$ membrane filtration, and the filter membranes with trapped MF were used for DNA extraction. The DNA was extracted using the ReliaPrep ${ }^{\mathrm{TM}}$ Blood DNA Miniprep System (Catalogue number A5082) according to the manufacturer's instructions [18] with some modifications.

Briefly, a volume of $180 \mu \mathrm{l}$ of phosphate-buffered saline was added to polycarbonate membranes with $\mathrm{MF}$ and incubated for 45 minutes while shaking ( $300 \mathrm{rpm})$. A volume of $20 \mu \mathrm{l}$ proteinase $\mathrm{K}$ and $200 \mu \mathrm{l}$ cell lysis buffer solution was added to the content and incubated at $56^{\circ} \mathrm{C}$ for 10 minutes to which $250 \mu$ l of binding buffer was added. The content was then transferred to ReliaPrep ${ }^{\mathrm{TM}}$ binding columns and centrifuged at $8,000 \mathrm{rpm}$. The binding columns were transferred to fresh collection tubes, and $500 \mu$ l of column wash solution was added and centrifuged for three minutes at $8,000 \mathrm{rpm}$ and the follow through was discarded. After repeating the former step twice, the binding columns were finally eluted with $50 \mu \mathrm{l}$ nuclease-free water. The DNA concentrations were measured by fluorometry according to the instructions (Manual \#TM396: http://www.promega.com/protocols/) and stored at $-20^{\circ} \mathrm{C}$ until used.

2.2. DNA Amplification. The procedure stated by Rishniw et al. was used with some modifications using Promega reagents [19]. The pan-filarial primers (DIDR-F1 5'-AGT GCG AAT TGC AGA CGC ATT GAG-3' and DIDR-R1 $5^{\prime}$-AGC GGG TAA TCA CGA CTG AGT TGA-3') that spanned the ITS2 of the rDNA designed by Rishniw et al. were employed to amplify the target DNA region. Known positive and negative controls were included in each PCR reaction. The PCR procedure consisted of an initial heating step at $94^{\circ} \mathrm{C}$ for 2 minutes, 32 cycles of denaturation at $94^{\circ} \mathrm{C}$ for 30 seconds, annealing at $58^{\circ} \mathrm{C}$ for 30 seconds, and extension at $72^{\circ} \mathrm{C}$ for 30 seconds and a final extension at $72^{\circ} \mathrm{C}$ for 7 minutes and a soak at $4^{\circ} \mathrm{C}$ in an Applied Biosystems 2720 Thermal Cycler (Thermo Fisher Scientific).

Discrimination of the six species was based on the size of the amplified PCR products. DIDR-F1 and DIDR-R1 primers amplified $484 \mathrm{bp}, 542 \mathrm{bp}, 578 \mathrm{bp}, 584 \mathrm{bp}, 615 \mathrm{bp}$, and $664 \mathrm{bp}$ products from Dirofilaria repens, Dirofilaria immitis, Acanthocheilonema reconditum, Acanthocheilonema dracunculoides, B. malayi, and B. pahangi, respectively [19]. 


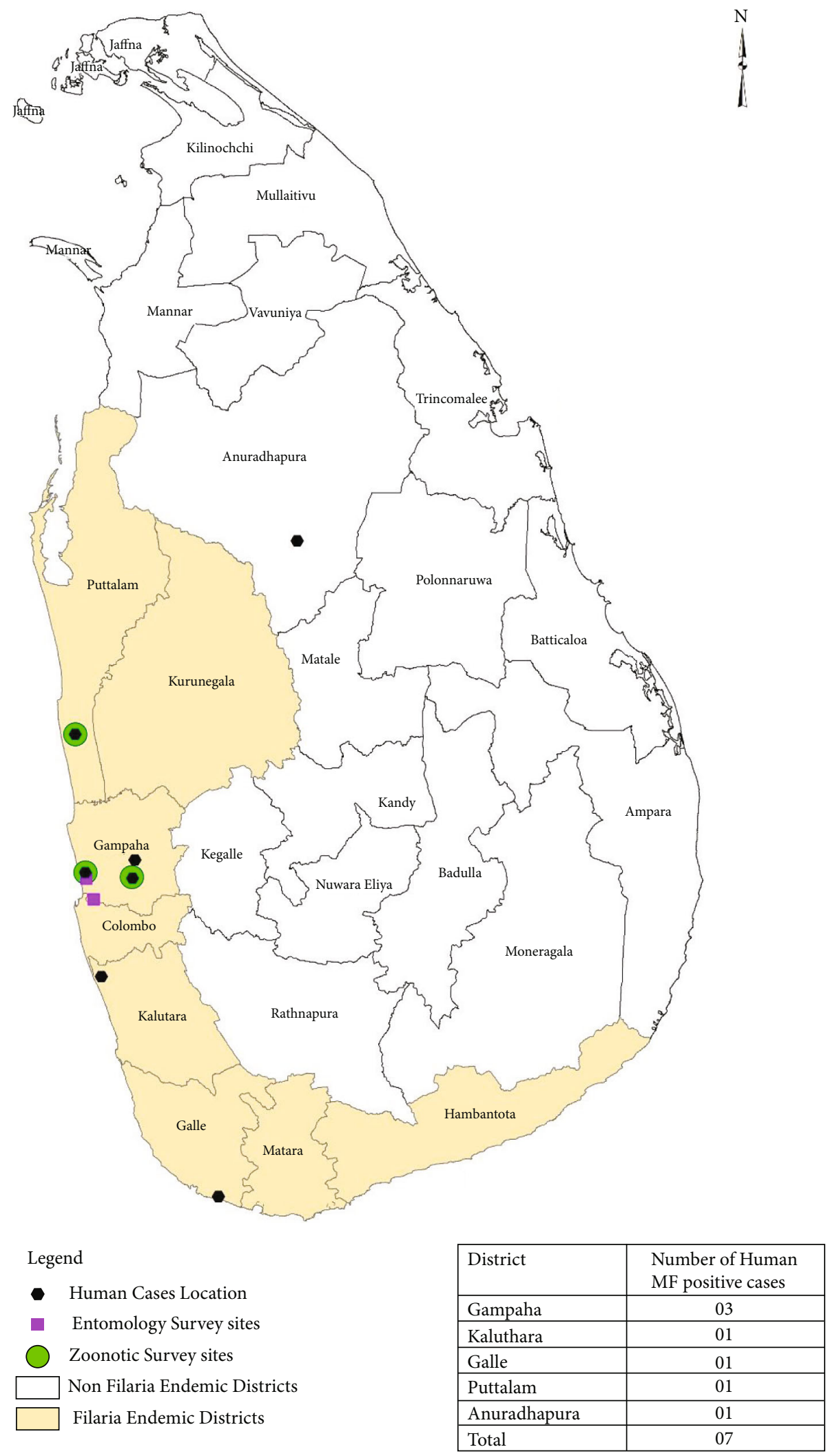

FIgURE 1: Human brugian cases and survey locations (Zoonotic and Entomology). 
2.3. Canine and Feline Sample Processing. The zoonotic surveillance for filarial parasites was done at three locations in the districts of Gampaha (Weliweriya and Wattala) and Puttalam (Madampe) of Western and Northwestern provinces, respectively (Figure 1). Molecular speciation of the zoonotic Brugia parasite by PCR using panfilarial primers specific for ITS2 region confirmed its identity as $B$. malayi as detailed in a previous publication (13).

\subsection{Mosquito Vector Analysis}

2.4.1. Sample Collection, DNA Extraction, and Amplification. The mosquito surveillance was carried out in the district of Gampaha in the same areas where zoonotic surveillance was done, using one cattle-baited net trap in each location (Figure 1). Mosquitoes of Mansonia species were identified using morphological keys. Heads and thoraces of all Mansonia species mosquitoes were dissected to identify filarial larvae.

DNA of filarial larvae was extracted using the MightyPrep reagent (Takara Bio Inc., Japan) according to the manufacturer's guide with some modifications as follows: the mosquito heads and thoraces that were positive for filarial larvae were crushed and mounted temporarily on glass slides and flushed with $200 \mu \mathrm{l}$ of MightyPrep reagent into a microcentrifuge tube. The lysate was homogenized by a hard vortex for 10 seconds. New pipette tips were used each time to prevent cross contamination of samples. The lysates were incubated at $95^{\circ} \mathrm{C}$ for 10 minutes. Subsequently, the sample lysates were cool down to room temperature. The cooled lysates were hard-vortexed for another 10 seconds. Finally, the samples were centrifuged at $12,000 \mathrm{rpm}$ for 10 minutes and stored at $-20^{\circ} \mathrm{C}$ until used for PCR. DNA amplification was done using the same set of primers and procedure used for human and animal samples.

2.4.2. DNA Sequencing. The Sanger method [20] was applied in cycle sequencing of amplicons (ITS2 region of rDNA) of MF in blood samples of humans $(n=2)$, canines $(n=3)$, and felines $(n=6)$. Sample selection was based on the concentration of the DNA extracts.

2.4.3. Homology Comparison. The forward and reverse chromatograms were assembled in Lasergene 8.00 software suite (DNASTAR Inc., USA). BLASTn tool was used to compare the nucleotide similarity of study sequences with those available in the NCBI GenBank database. Consensus sequences were aligned using MAFFT 7, and phylogenetic tree was developed using the Neighbor-Joining (NJ) algorithm executed in MEGA-X software [21], and the tree was constructed with K2P substitution model and gamma distributed rates. Bootstrap analysis of 1,000 replicates was performed to determine the robustness of clades. To develop the tree, 22 reference DNA sequences were retrieved from the GenBank database. Meantime, pairwise distances were calculated between individuals in each species using the MEGA-X software. Further, polymorphisms in the alignment were analyzed using the DnaSP (version 6.12.01 x64) software.
2.5. Ethical Clearance. Ethical approval for the study was obtained from the Ethics Review Committees of the Faculty of Medicine University of Kelaniya (P/108/09/2016) and the Medical Research Institute (40/2016).

\section{Results}

Six of the seven human blood samples were positive for Brugia sp. MF by NBS that were tested by PCR produced a band of $615 \mathrm{bp}$ specific for B. malayi (Figure 2). The sample that failed to elicit a PCR band had very low microfilaraemia (1MF/slide). All human blood samples that were only positive for anti-Brugia antibodies by BRT $(n=9)$ failed to elicit a PCR band. The zoonotic samples also elicited bands of $615 \mathrm{bp}$ specific for B. malayi (Figure 3).

A total of 82 Mansonia sp. mosquitoes were identified in the cattle-baited net trap collection, namely, $M$. annulifera (65), M. uniformis (14), and M. indiana (3). Filarial larval stages were detected in $20.73 \%$ (17/82) of the dissected Mansonia mosquitoes. Of them, $7.14 \%(1 / 14)$ and $24.6 \%(16 / 65)$ were $M$. uniformis and $M$. annulifera, respectively. The DNA extracts of all infected Mansonia mosquitoes elicited the $615 \mathrm{bp}$ band of $B$. malayi when analysed by pan-filarial primer-specific PCR.

\section{Phylogenetic Analysis}

Homology analysis performed on GenBank using the BLASTN of NCBI and the amplified ITS2 sequences of the B. malayi MF isolated from humans, dogs, and cats in this study showed the sequences to have higher homology with B. pahangi than B. malayi (Figure 4). Homology analysis of developed sequences from different host species was compared, and the highest estimated evolutionary divergence of B. malayi MF was observed between dogs and cats $(2.7 \%)$ and the lowest between humans and cats $(0.7 \%)$. The estimated evolutionary divergence of $B$. malayi MF specimens between humans and dogs was 1.9\% (Annex 1).

Phylogenetic analyses of the generated ITS2 gene sequences from human, canine, and feline blood samples were clustered with B. pahangi and B. malayi. All the Brugia sp. ITS2 sequences demonstrated a monophyletic origin from the same ancestor and formed separate clusters (Figure 4). The lengths of the horizontal lines are proportional to the minimum number of nucleotide differences required to join nodes. The mean K2P divergence analysis of the new ITS2 sequences showed less than 3\% phylogenetic divergence with other studied brugian sequences (Table 1). The interspecies K2P divergence between new sequences and B. malayi was $2.41 \%$ while that of for B. pahangi is $1.8 \%$.

During the polymorphism analysis, 21 parsimony informative sites with one deletion were identified and nine (09) of them were crucial polymorphic sites in the ITS2 gene alignment (Table 2). The results indicated that the new sequences were similar to both $B$. pahangi and $B$. malayi. This is similar to the Neighbor-Joining phylogenetic tree inferred using the Tajima-Nei method with 500 replicates bootstrap support. The new sequences exhibited similarities to B. malayi in 277 and 288 positions while showing 


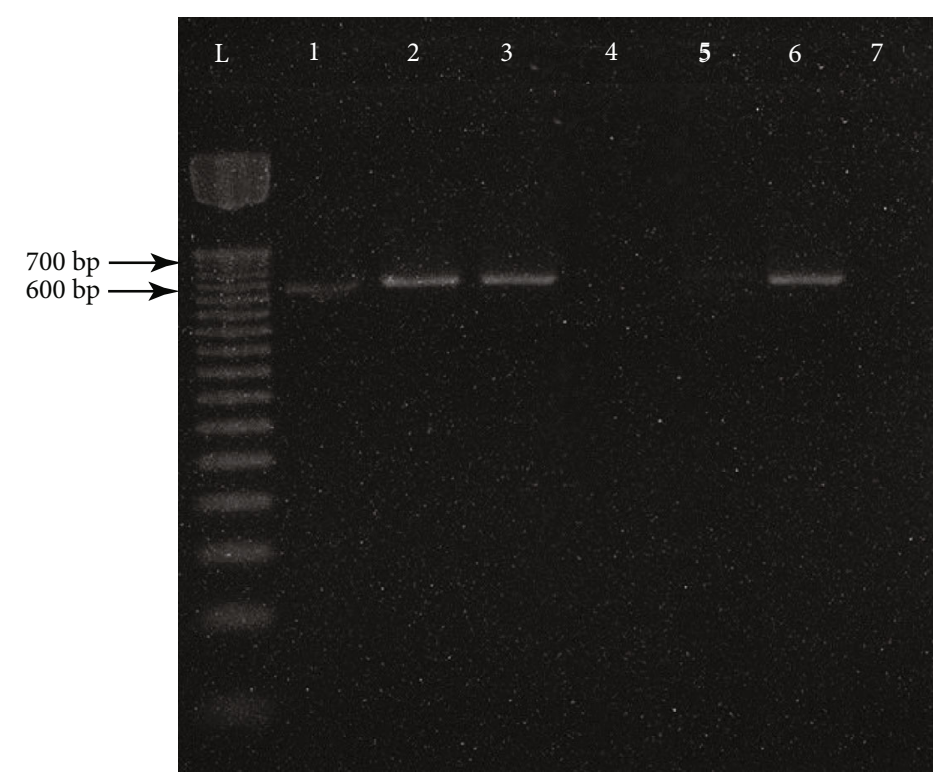

FIGURE 2: Gel electrophoresis of the PCR products of human samples. The gel image shows the results of the PCR amplification of samples 1 to 7 using Pan-filarial primers (Lanes 1 to 5: human samples, Lane 6: positive control, Lane 7: negative control, and Lane L: 50 bp DNA marker).

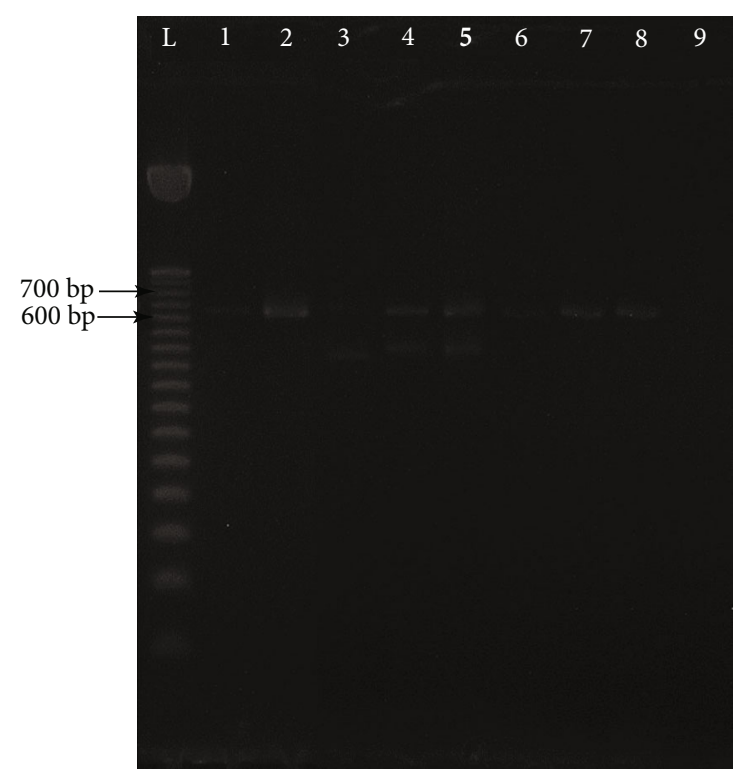

FIGURE 3: Gel electrophoresis of pan-filarial primer-specific PCR amplicons of human, canine, and feline samples from the zoonotic study area Pubudugama. The gel image shows the results of the pan-filarial primer-specific PCR amplification of samples 1 to 9 (Lanes 1: human sample, Lane 2 to 4 : dog samples, Lane 5 to 7: cat samples, Lane 8: positive control, Lane 9: negative control, and Lane L: 50 bp DNA marker).

differences at A99T and A118G with 176delA showing similarities to $B$. pahangi. However, the new sequences also demonstrated fixed substitutions at A36T, A296G, T373A, and G482A which made the new sequences different from $B$. pahangi. Therefore, the new sequences may probably be a variant or hybrid strain of both B. malayi and B. pahangi.

\section{Discussion}

PCR confirmed that the reemerged subperiodic Brugia parasite was the same as that circulating among dogs and cats in the region. The phylogenetic analysis of the ITS2 region of the rDNA of both human and zoonotic parasites indicated that the reemerged B.malayi parasites had a close resemblance to $B$. pahangi. However, the $\mathrm{K} 2 \mathrm{P}$ distance between the new sequences and available previous sequences was less than 3\%, the cut-off for nematode species demarcation $[22,23]$.

The presence of a novel zoonotic Brugia strain closely related to $B$. malayi and $B$. pahangi has been previously queried on molecular characterization of the rDNA sequences of a filarial nematode and comparison with known gene sequences in the GenBank. These filarial nematodes were isolated from the inguinal lymph nodes of a patient based in the city of New York with an extensive travel history to Central America and the Caribbean [24].

A canine survey in Kerala had reported the presence of $B$. malayi like MF that had elicited a histochemical staining pattern consistent with that of B. malayi. The Hhal primer PCR product of this $B$. malayi like parasites was cloned and sequenced (2 clones, Accession numbers JN 601136 and JN 601137), and the phylogeny revealed that the $B$. malayi like parasite was genetically closer to $B$. pahangi suggesting the existence of a genetic variant closely related to $B$. malayi and B. pahangi in the natural environment [25].

Further, the new sequences demonstrated $2.4 \%$ and $1.8 \%$ genetic divergence with $B$. malayi and B. pahangi, respectively. This interspecies genetic distance is much higher than the distance difference between $B$. malayi and B. pahangi, which is $0.5 \%$. Similar low levels of interspecies divergence have been reported previously in ITS-2-based 


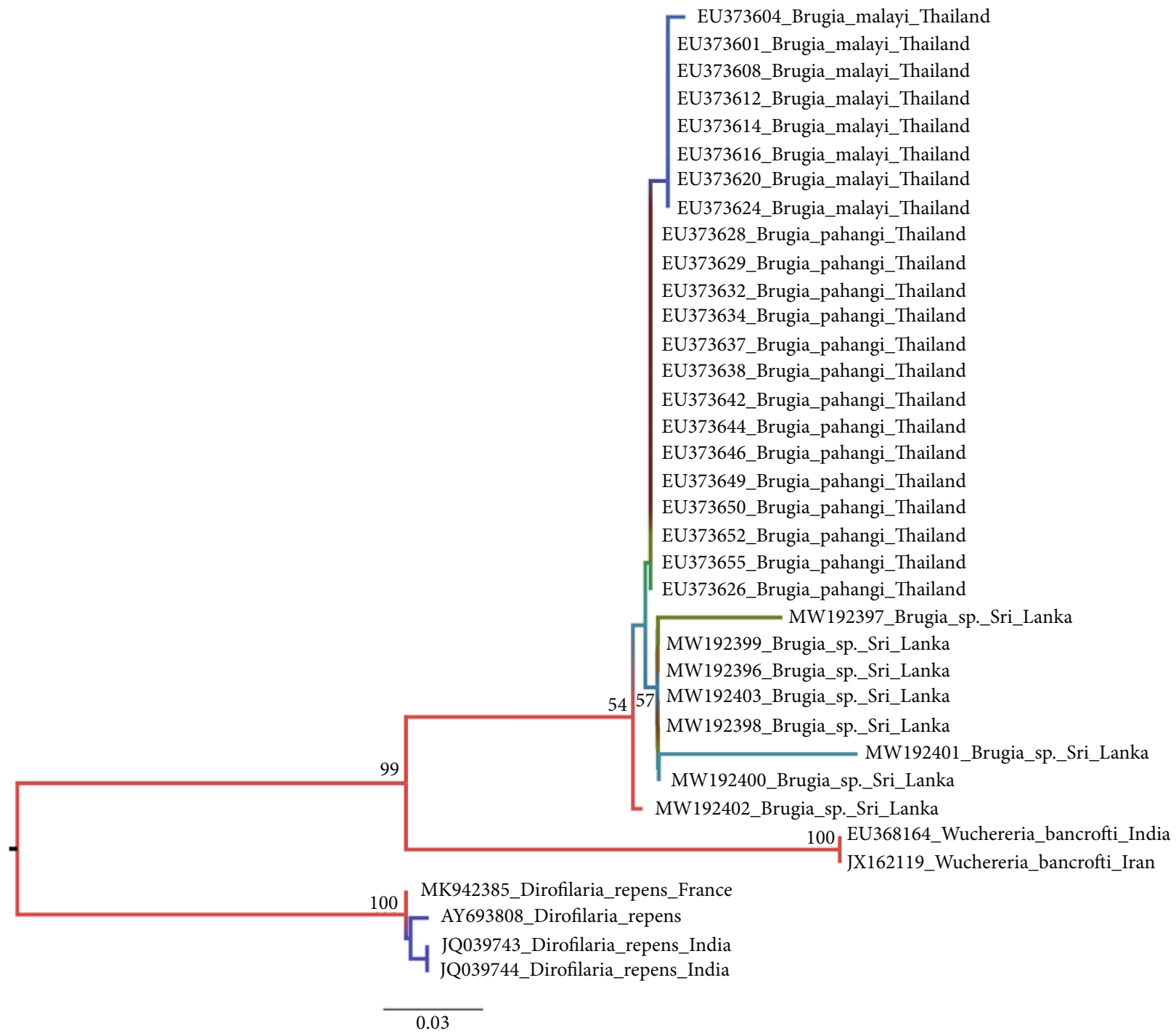

FIGURE 4: Phylogenetic tree constructed from partial rDNA sequencing (ITS2 region); 14 sequences of $B$. pahangi and 8 sequences of $B$. malayi were retrieved from GenBank and are shown with accession numbers. Phylogenetic tree was constructed using MEGA-X software with 1,000 bootstrap support (MW192396-8-Cat, Madampe; MW192399-Human, Pubudugama; MW192400-Human, Weliweriya; MW192401-Dog, Pubudugama; MW192402-3-Cat, Pubudugama).

TABLE 1: Interspecific genetic distances among the analysed species.

\begin{tabular}{lccccc}
\hline & SL_G1 & B. malayi & B. pahangi & W. bancrofti & D. repens \\
\hline SL_G1 & 0.0 & & & & \\
B. malayi & 2.414 & 0.0 & & & \\
B. pahangi & 1.801 & 0.589 & 0.0 & & \\
W. bancrofti & 22.53 & 21.074 & 20.309 & 0.0 & \\
D. repens & 33.359 & 31.996 & 31.113 & 37.115 & 0.0 \\
\hline
\end{tabular}

studies of hookworms from northern fur seals and California sea lions [23].

The possibility of this variant strain of $B$. malayi being the species documented as $B$. ceylonensis requires due consideration. The adult filarial nematodes of $B$. ceylonensis were first described in 1962 in the lymphatic glands of dogs from Sri Lanka. It was documented as a novel species closely related to $B$. patei based on morphological characteristics [26]. However, genomic data of $B$. ceylonensis is not available in GenBank database for comparison with the reemerged B. malayi.

The results of the present study indicate that the variant strain is well established among animals with occurrence of spill-over infections among humans. This variant B. malayi appears to have a limited capacity to infect humans as case numbers were relatively low compared to the heavy zoonotic reservoirs of infection in Sri Lanka [13]. This could be attributed to low transmissibility owing to vector characteristics (low affinity of Mansonia sp. mosquitoes for human blood) or the enhanced immune response generated by a poorly adapted zoonotic parasite conferring natural resistance to infection. The rising number of cases in the recent past may be indicative of the parasite's potential to evolve and adapt to humans or may reflect the research interest generated by the PELF. 
TABLE 2: Crucial polymorphic sites observed in the study sequences.

\begin{tabular}{lccc}
\hline Position & Study sequences & B. malayi & B. pahangi \\
\hline 36 & T & A & A \\
99 & T & A & T \\
118 & G & A & G \\
176 & - & A & - \\
277 & T & T & - \\
282 & G & G & A \\
296 & G & A & A \\
373 & T & A & A \\
482 & G & A & A \\
\hline
\end{tabular}

Sentinel surveillance of animals and xenomonitoring may serve in defining populations at risk of infection. Thus, vector identification is important not only for implementing the appropriate vector control measures but also for mapping the distribution of infection. M. annulifera and $M$. uniformis are zooanthropophagic mosquito species in Sri Lanka that were implicated in the transmission of periodic $B$. malayi in the past $[27,28]$. This study confirmed their capability of transmitting the variant B. malayi (subperiodic) in Sri Lanka.

ITS regions 1 and 2 have been used by many investigators for studies on phylogenetic reconstruction, genetic variability, and divergence of closely related taxa of a wide range of organisms [29-31]. The molecular characterization of the MF of this variant B. malayi isolated from humans was limited to two samples, and analysis was focused only on the ITS2 region of the rDNA. A more comprehensive genomewide analysis of rDNA as well as mitochondrial DNA of MF and adult stages of this variant B. malayi may be required for clarification of the taxonomy of the parasite. Further studies on B. malayi parasites isolated from humans and animals from different geographical locations in the country as well as comprehensive entomological surveys which cover a wide array of mosquito species are indicated to characterize the novel variant zoonotic $B$. malayi.

\section{Conclusion}

The reemerged B. malayi parasite in Sri Lanka appears to be a novel genetic variant. Domestic canines and felines were identified as the zoonotic reservoirs and Mansonia sp. mosquitoes (M. annulifera and M. uniformis) were implicated as vectors. This reemerged parasite could well pose a threat to the LF elimination status of the country because vectors and infectious zoonotic reservoirs are present in abundance. The application of molecular identification techniques will be invaluable for clarification of taxonomy, epidemiology, and ecology of this novel variant strain of $B$. malayi.

\section{Data Availability}

The datasets supporting the conclusions of this article are included in the manuscript.

\section{Conflicts of Interest}

The authors declare that they have no conflict of interests.

\section{Acknowledgments}

This project was funded by the "Strengthening Research2016" (RP/03/SR/04/06/01/2016) grant of the University of Kelaniya. The authors wish to acknowledge the support extended by Mr. Tharaka Wijerathna, Mr. Lalinka Herath in developing the phylogeny and production of visual images, and Dr. P. A D. H. N. Gunathilaka for providing arthropod DNA extraction kits.

\section{Supplementary Materials}

Aneex 1 Homology analysis of developed sequences from different host species. (Supplementary Materials)

\section{References}

[1] World Health Organization, "Lymphatic filariasis," 2020, https://www.who.int/en/news-room/fact-sheets/detail/ lymphatic-filariasis/.

[2] P. C. Beaver and T. C. Orihel, "Human infection with filariae of animals in the United States," The American Journal of Tropical Medicine and Hygiene, vol. 14, no. 6, pp. 1010-1029, 1965.

[3] K. Chansiri, T. Tejangkura, P. Kwaosak, N. Sarataphan, S. Phantana, and W. Sukhumsirichart, "PCR based method for identification of zoonostic _Brugia malayi_microfilariae in domestic cats," Molecular and Cellular Probes, vol. 16, no. 2, pp. 129-135, 2002.

[4] K. Kanjanopas, W. Choochote, A. Jitpakdi et al., "Brugia malayi in a naturally infected cat from Narathiwat Province, southern Thailand," The Southeast Asian Journal of Tropical Medicine and Public Health, vol. 32, no. 3, pp. 585-587, 2001.

[5] T. C. Orihel and M. L. Eberhard, "Zoonotic filariasis," Clinical Microbiology Reviews, vol. 11, no. 2, pp. 366-381, 1998.

[6] L. H. Tan, M. Y. Fong, R. Mahmud, A. Muslim, Y. L. Lau, and A. Kamarulzaman, "Zoonotic Brugia pahangi filariasis in a suburbia of Kuala Lumpur City, Malaysia," Parasitology international, vol. 60, no. 1, pp. 111-113, 2011.

[7] M. L. Eberhard, L. J. DeMeester, B. W. Martin, and P. J. Lammie, "Zoonotic Brugia infection in western Michigan," The American Journal of Surgical Pathology, vol. 17, no. 10, pp. 1058-1061, 1993.

[8] U. Schweinfurth, "Filarial diseases in Ceylon: a geographic and historical analysis," Ecology of Disease, vol. 2, no. 4, pp. 309319, 1983.

[9] World Health Organization, "WHO officially declares Sri Lanka filariasis free," 2016, http://origin.searo.who.int/ srilanka/documents/WHO_officially_declares_Sri_Lanka_ filariasis_free/en/.

[10] N. T. Chandrasena, R. Premaratna, D. S. Samarasekera, and N. R. de Silva, "Surveillance for transmission of lymphatic filariasis in Colombo and Gampaha districts of Sri Lanka following mass drug administration," Transactions of the Royal Society of Tropical Medicine and Hygiene, vol. 110, no. 10, pp. 620-622, 2016. 
[11] P. Fischer, I. Bonow, T. Supali, P. Rückert, and N. Rahmah, "Detection of filaria-specific IgG4 antibodies and filarial DNA, for the screening of blood spots for Brugia timori," Annals of Tropical Medicine and Parasitology, vol. 99, no. 1, pp. 53-60, 2005.

[12] C. H. Mallawarachchi, T. G. A. Nilmini Chandrasena, R. Premaratna, S. M. N. S. M. Mallawarachchi, and N. R. de Silva, "Human infection with sub-periodic Brugia spp. in Gampaha District, Sri Lanka: a threat to filariasis elimination status?," Parasites \& Vectors, vol. 11, no. 1, p. 68, 2018.

[13] C. H. Mallawarachchi, N. T. G. A. Chandrasena, S. Wickramasinghe et al., "A preliminary survey of filarial parasites in dogs and cats in Sri Lanka," PLoS One, vol. 13, no. 11, article $\mathrm{e} 0206633,2018$.

[14] R. P. Rajapakshe, W. S. Perera, R. L. Ihalamulla et al., "Study of dirofilariasis in a selected area in the Western Province," The Ceylon Medical Journal, vol. 50, no. 2, pp. 58-61, 2005.

[15] J. R. Palmieri, S. Ratiwayanto, S. Masbar, S. Tirtokusumo, J. Rusch, and H. A. Marwoto, "Evidence of possible natural infections of man with Brugia pahangi in South Kalimantan (Borneo), Indonesia," Tropical and geographical medicine, vol. 37, no. 3, pp. 239-244, 1985.

[16] M. Y. Fong, R. Noordin, Y. L. Lau, F. W. Cheong, M. H. Yunus, and Z. M. Idris, "Comparative analysis of ITS1 nucleotide sequence reveals distinct genetic difference between Brugia malayi from Northeast Borneo and Thailand," Parasitology, vol. 140, no. 1, pp. 39-45, 2013.

[17] A. E. Paniz Mondolfi and E. M. Sordillo, "Invited editorial: zoonotic lymphatic filariasis in the Americas: trends in epidemiology, diagnosis and treatment, with special emphasis on brugian filariasis," Recent Patents on Anti-Infective Drug Discovery, vol. 9, no. 3, pp. 161-163, 2014.

[18] Promega Corporation, "ReliaPrep Blood gDNA Miniprep System," 2012.

[19] M. Rishniw, S. C. Barr, K. W. Simpson, M. F. Frongillo, M. Franz, and J. L. Dominguez Alpizar, "Discrimination between six species of canine microfilariae by a single polymerase chain reaction," Veterinary Parasitology, vol. 135, no. 3-4, pp. 303-314, 2006.

[20] F. Sanger, S. Nicklen, and A. R. Coulson, "DNA sequencing with chain-terminating inhibitors," Proceedings of the National Academy of Sciences of the United States of America, vol. 74, no. 12, pp. 5463-5467, 1977.

[21] S. Kumar, G. Stecher, M. Li, C. Knyaz, and K. Tamura, "MEGA $\mathrm{X}$ : molecular evolutionary genetics analysis across computing platforms," Molecular Biology and Evolution, vol. 35, no. 6, pp. 1547-1549, 2018.

[22] H. H. Mejía-Madrid, "Soil nematode abundance and diversity from four vegetation types in Central Mexico," Nematology, vol. 20, no. 1, pp. 15-32, 2017.

[23] S. Nadler, "Species delimitation and nematode biodiversity: phylogenies rule," Nematology, vol. 4, no. 5, pp. 615-625, 2002.

[24] A. E. Paniz-Mondolfi, T. Gárate, C. Stavropoulos et al., "Zoonotic filariasis caused by novel Brugia sp. nematode, United States, 2011," Emerging infectious diseases, vol. 20, no. 7, pp. 1248-1250, 2014.

[25] R. Ravindran, S. Varghese, S. N. Nair et al., "Canine filarial infections in a human Brugia malayi endemic area of India," BioMed Research International, vol. 2014, Article ID 630160, 9 pages, 2014.
[26] L. G. Jayewardene, "On two filarial parasites from dogs in Ceylon,Brugia ceylonensisn.sp. andDipetalonemasp.inq," Journal of Helminthology, vol. 36, no. 3, pp. 269-280, 1962.

[27] A. S. Dissanaike, W. Abeyewickreme, M. D. Wijesundera, M. V. Weerasooriya, and M. M. Ismail, "Human dirofilariasis caused by Dirofilaria (Nochtiella) repens in Sri Lanka," Parassitologia, vol. 39, no. 4, pp. 375-382, 1997.

[28] S. N. McNulty, M. Mitreva, G. J. Weil, and P. U. Fischer, "Inter and intra-specific diversity of parasites that cause lymphatic filariasis," Infection, Genetics and Evolution, vol. 14, pp. 137146, 2013.

[29] M. De Rojas, M. Mora, J. Ubeda, C. Cutillas, M. Navajas, and D. Guevara, "Phylogenetic relationships in rhinonyssid mites (Acari: Rhinonyssidae) based on ribosomal DNA sequences: insights for the discrimination of closely related species," Parasitology Research, vol. 88, no. 7, pp. 675-681, 2002.

[30] R. Morales-Hojas, R. A. Cheke, and R. J. Post, "A preliminary analysis of the population genetics and molecular phylogenetics of Onchocerca volvulus (Nematoda: Filarioidea) using nuclear ribosomal second internal transcribed spacer sequences," Memórias do Instituto Oswaldo Cruz, vol. 102, no. 7, pp. 879-882, 2007.

[31] Z. T. Maafi, S. Subbotin, and M. Moens, "Molecular identification of cyst-forming nematodes (Heteroderidae) from Iran and a phylogeny based on ITS-rDNA sequences," Nematology, vol. 5, no. 1, pp. 99-111, 2003. 